in perivascular spaces. Many remaining neurones contained one or more spherical concentric hyaline inclusion bodies of Lewy. Ultrastructurally these consisted of fibrillary and granular material with the fibrils more abundant at the periphery where they tended to be radially arranged. The granular component usually predominated in the centre where it formed a compact mass corresponding with the refractile and strongly acidophilic central core. Some Lewy bodies appeared larger and more homogeneous without any central core and these were composed entirely of uniformly dispersed fibrillary material. There was no evidence from electron microscopy that altered melanin granules entered into the composition of the inclusions, but occasionally degenerating organelles, particularly mitochondria, were seen in these structures, suggesting that they represented areas of cytoplasmic degeneration within the neurones.

Corpora amylacea, frequent in the substantia nigra of these cases, differed from the Lewy bodies both in situation and in ultrastructure, being composed of larger, coarser granules and fibrils partially enclosed by a limiting membrane. Although there was reduction in number of melanin granules in Parkinsonism no significant structural difference in the granules was found on electron microscopy in comparison with those from normal control material of similar age. Although chemically different, the ultrastructural similarity between melanin and lipofuscin was striking.

\section{A CLINICO-PATHOLOGICAL AND FAMILY STUDY OF POLYCYSTIC DISEASE OF THE KIDNEYS AND LIVER IN CHILDREN}

H. M. BLYTH AND B. G. OCKENDEN (M.R.C. Clinical Genetics Unit and Departmen: of Morbid Anatomy, Institute of Child Health, Guilford Street, London) The purpose of this study was to define those types of cystic disease of the kidneys occurring in children which carry a high risk of recurrence within the family. Difficulty had been experienced with genetic counselling for these conditions.

Index patients, having cystic malformation of the renal tubules and intrahepatic bile ducts (polycystic disease of the kidneys and liver), were ascertained ( $a$ ) from surgical and postmortem material examined at The Hospital for Sick Children and (b) from families referred to the Genetic Clinic at the hospital over the past 20 years. The latter were included only if the diagnosis could be confirmed histologically in at least one member of the family.

Twenty-nine cases in 24 families were studied in which the diagnosis was confirmed histologically; in three of these families a further six relatives have been included on clinical and/or radiological grounds.

Dominant inheritance is certain in one family and three others come into this 'adult' type on the histological criteria of focal cystic malformation of renal tubules and hepatic bile ducts.

In 20 families an autosomal recessive pattern of inheritance is indicated. In this 'childhood' type the cystic malformation affects all the intrahepatic bile ducts and the renal tubular lesions are uniformly dispersed throughout both kidneys. On clinico-pathological grounds the 25 cases in these families fell into four contiguous groups. The most easily recognized groups were those presenting earliest in the perinatal and neonatal periods, with bilateral large kidneys. A con- $\vec{P}$ trasting group presents in childhood with hepatomegaly을 and portal hypertension. Between these is a group $\bar{\sigma}$ presenting in infancy with hepatosplenomegaly and $\overline{\frac{\sigma}{\sigma}}$ developing renal insufficiency later. Within individual $\mathbb{D}$ families the type of disorder breeds true, suggesting that each group represents a recessive condition, but that a $\infty$ different mutant gene is involved in each group.

This paper will be published in full.

\section{A CASE OF FATAL CLOSTIDIUM WELCHII TOXAEMIA DUE} TO WARD INFECTION

J. G. ALEXANDER (Royal Infirmary, Hull) A 14-day-old male baby had a small sacral meningocoele repaired in $\vec{G}$ a neurosurgery theatre. There was no adrenalin in the local anaesthetic. He collapsed 20 hours later with meningitis due to Clostridium welchii (toxin type $\mathrm{A}$ 응 serologically untypable). Intravenous ampicillin and cloxacillin did not prevent death from acute haemolytic $\vec{c}$ anaemia 27 hours after operation. The organism was not present in his faeces. Histological examination of the wound edges showed morphological Clostridium welchii. From a large number of swabs from the theatre suite, the ward, and the portable incubator in which the baby $\square$ travelled from (but not to) the theatre, there were seven toxin type A serologically untypable strains isolated. A rabbit antiserum made against the baby's strain agglut- $\bar{O}$ inated two strains from the incubator and one from the

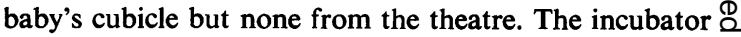
was not infected from the baby because of an imperme- $\overrightarrow{\vec{F}}$ able Nobecutane plastic film over the wound (causing $\frac{0}{3}$ tissue anaerobiosis). Previously, patients whose wounds yielded Clostridium welchii had not shown toxicity. Such a patient was the incubator's previous occupant and her Clostridium welchii had not been kept. This (or another ward strain) must have got on to the patient's skin in the $\bar{\sigma}$ ward. The bath used to bathe him preoperatively had 3 been previously wiped with Savlon. Three applications $\dot{\partial}$ of $0.5 \%$ chlorhexidine in $70 \%$ spirit to the operation area in the theatre did not prevent the infection. The local anaesthetic, Nobecutane spray, and dressings were sterile. Clostridium welchii strains were not found in the $D$ theatre air inlet ventilators or anywhere in the neurosurgery theatre.

\section{SIGNIFICANCE OF THE GIANT ORTHOCHROMATIC ERYTHROBLAST}

H. B. GOODALl (Royal Infirmary, Dundee) The giant orthochromatic erythroblast is a greatly enlarged poly-

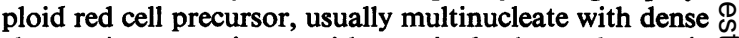
chromatin, sometimes with a single hyperchromatic? nuclear mass and with mature, well haemoglobinized cytoplasm. This characteristic morphological change may occur in the bone marrow in a wide range of clinical circumstances: as an inborn defect associated with ineffec- $\mathbb{D}$ tive erythropoiesis; as an accompaniment of neoplasia $\sigma$ of erythroblasts or lymphoreticular cells; in deficiency of 